\title{
A!
}

This is an electronic reprint of the original article.

This reprint may differ from the original in pagination and typographic detail.

Zubiaga, A.; Ervasti, M. M.; Makkonen, I.; Harju, A.; Tuomisto, F.; Puska, M. J.

\section{Modeling positronium beyond the single particle approximation}

Published in:

Journal of Physics B: Atomic Molecular and Optical Physics

DOI:

10.1088/0953-4075/49/6/064005

Published: 29/02/2016

Document Version

Peer reviewed version

Please cite the original version:

Zubiaga, A., Ervasti, M. M., Makkonen, I., Harju, A., Tuomisto, F., \& Puska, M. J. (2016). Modeling positronium beyond the single particle approximation. Journal of Physics B: Atomic Molecular and Optical Physics, 49(6), 113. [064005]. https://doi.org/10.1088/0953-4075/49/6/064005

This material is protected by copyright and other intellectual property rights, and duplication or sale of all or part of any of the repository collections is not permitted, except that material may be duplicated by you for your research use or educational purposes in electronic or print form. You must obtain permission for any other use. Electronic or print copies may not be offered, whether for sale or otherwise to anyone who is not an authorised user. 


\title{
Modeling positronium beyond the single particle approximation
}

\author{
A. Zubiagał, M. M. Ervasti, I. Makkonen, A. Harju, F. \\ Tuomisto, and M. J. Puska \\ Department of Applied Physics, Aalto University School of Science, FI-00076 \\ AALTO, Espoo, Finland \\ January 2016
}

\begin{abstract}
Understanding the properties of the positronium atom in matter is of interest for the interpretation of positron annihilation experiments. This technique has a unique capability for the investigation of nanometer sized voids and pores soft molecular materials (polymers, liquids or biostructures) and porous materials. However, detailed interpretations of the experimental data rely on modeling of the annihilation properties of positronium in the host material. New applications of the technique are being developed but the computational models still are based on single particle approaches and there is no way to address the influence of the electronic properties of the host material. In this work we discuss new directions of research.
\end{abstract}

Submitted to: J. Phys. B: At. Mol. Opt. Phys.

\section{Introduction}

Positronium (Ps) is the bound state of an electron and a positron. Inside materials, Ps forms mostly in molecular matter, in some insulators such as $\mathrm{SiO}_{2}$ or in porous materials like zeolites or metal-organic frameworks. Upon implantation of a positron in a material, Ps forms in regions of low electron density [1], whereas a screening cloud of electrons surrounds the positron in metals and semiconductors.

Ps is produced by the capture of an electron of the host material by the positron or by the binding of a free electron from the implantation track blob in both molecular (polymers, liquids) and porous materials. The capture of an electron from a host material is subject to the Ore gap which limits the capacity for creation of Ps to positrons within an energy range limited by the ionization energy of the medium $(I)$ and the binding energy of $\mathrm{Ps}_{\mathrm{S}}\left(E_{P s}\right)$ as

$I>E>I-E_{P s}$.

$\ddagger$ Current address: Institute for Chemical and Bioengineering, ETH Zurich, Vladimir-Prelog-Weg 1, CH-8093 Zurich, Switzerland 
Positrons with energy larger than $I$ will result in high energy Ps which will probably dissociate in subsequent collisions. On the other hand, positrons with lower energy cannot ionize the medium.

Inside the blob composed of free electrons and radicals ionized during the slowdown, the positron can bind a free electron and escape the blob as a quasi-thermalized Ps. The formation of Ps depends on the electron affinity of the host material, the kinetics of the electron-radical recombination, and the screening of the electron-positron attraction by the surrounding media $[1,2]$.

The relevance of thermalized Ps for material characterization comes from its ability to localize at open volume pockets such as large vacancies or voids where the Ps-matter repulsion is minimum. In vacuum, o-Ps (ortho-Ps, the spin-triplet state) has a relatively long lifetime of 142 ns because the fast self-annihilation of para-Ps (the spin-singlet state) via the fast two-gamma channel is prohibited for o-Ps by the conservation of the angular momentum. Inside matter, the pick-off annihilation with an electron of the host material through the two-gamma channel reduces the o-Ps lifetime typically to 1-100 ns depending on the local electron density and size of the open volume pocket [3]. The electron in o-Ps hinders efficiently further screening by electrons in the matter and prevents large values of the overlap. Scattering experiments of Ps off noble gas atoms and light closed-shell molecules has shown to be dominated by the repulsive electronelectron interaction at short distances [4]. Ps does not usually form bound states with single molecules, except at longer separations where the dispersion (van der Waals) attraction may play a role especially for molecules of high polarizability. The resulting pick-off annihilation rate remains however smaller than in metals and semiconductors and it depends weakly on the electronic properties of the host material.

The combination of the strong tendency to localize in open volume and the strong dependence of the annihilation rate on the size of the open volume pocket makes oPs an excellent probe for nano- and meso-sized pores. When interacting with radicals or atoms with unpaired electrons, o-Ps can form a strong chemical bond (chemical quenching) or become a para-Ps ( $\mathrm{p}-\mathrm{Ps}$ ) spin-singlet (spin conversion). In both cases the lifetime of o-Ps is dramatically reduced below $1 \mathrm{~ns}$. The experimental signature of the pick-off annihilation of o-Ps can thus be separated from other annihilation processes where the positron interacts stronger with the electrons of matter. Positronium annihilation lifetime spectroscopy exploits this property to measure the distribution of the open volume in materials such as porous $\mathrm{SiO}_{2}[5,6]$, zeolites $[7,8]$, graphene oxide composites [9], metal-organic frameworks [10], polymers [11, 12] or biostructures [13, 14]. Recently, Milina et al . applied Ps advanced lifetime decomposition methods to hierarchical zeolites and showed the correlation between the catalytic performance and the fraction of o-Ps annihilating in vacuum [8].

The analysis of positron annihilation spectroscopy experiments benefit from predictions by computational models. For positrons in metals and semiconductors, the quantum-mechanical state of a single positron can be calculated based on the results of many-body theories for a delocalized positron in a homogeneous electron gas $[15,16]$. 
Recent advances in the application of the impulse approximation have been able to explain the electron-like scattering properties of Ps scattering off noble atoms and small molecules [17]. However, an ab-initio treatment of a system comprising two lightparticle species forming a Ps bound state inside a host material remains beyond the presently available computing capacity. The treatment of the dynamical correlations remains being a major problem when the adiabatic approximation, which allows treating classically the atom nuclei, cannot be applied.

This work explores two approaches to simulate and understand systems involving Ps beyond the single particle approach. Our aim is to develop calculation schemes able to predict accurate pick-off annihiation rates using atomistic models for relevant materials. Sections 2 and 3 of the present paper review the development of full-correlation potentials from accurate many-body explicitly correlated gaussians (ECG) calculations and apply them to obtain accurate Ps pick-off annihilation rates in molecular soft matter. Section 4 discusses the possibility of analyzing correlated states involving positronium using ideas based on reduced density matrices and natural geminals.

\section{Simulation of Ps in molecular matter}

\subsection{Single particle models}

An attractive starting point for modeling $P_{s}$ is to consider it as a distinguishable particle. This is in line with the view of a long-living o-Ps in open volume pockets interacting weakly with surrounding matter and annihilating through the pick-off process.

A semi-empirical model widely used to describe Ps in small pores and voids $(<2$ $\mathrm{nm}$ ) is that developed by Tao [19] and parametrized first by Eldrup et al . [20]. The model relates the Ps lifetime to the pore/void radius. Ps is considered as a single particle and the pore/void is modeled using a spherical well. Extensions have been developed for rectangular or elliptic potential wells [21] and for large voids where Ps can annihilate in excited states. [22, 5, 23] The electron density of the material forms a layer of thickness $\Delta \mathrm{R}$ on the surface of the well. The pick-off annihilation rate $\left(\lambda_{T E}^{p o}\right)$ is calculated as the overlap of the densities of the single particle and the electrons of the material. For a spherical void of radius $R$, it is

$\lambda_{T E}^{p o}=2.0\left(\mathrm{~ns}^{-1}\right)\left[1-\frac{R}{R+\Delta R}+\frac{1}{2 \pi} \sin \left(\frac{2 \pi R}{R+\Delta R}\right)\right]$.

Interestingly a classical model proposed by Wada et al . [24] reproduces the values predicted by the extended Tao-Eldrup model. Unfortunately, the parameter of the model, $\Delta \mathrm{R}$, is not well known what limits its ability to describe the chemical dependencies of annihilation properties. Anisotropic pore shapes and non-homogeneous

environment, like biostructures or materials composed by polar molecules need also more detailed description of the Ps-matter interaction.

Other models have been proposed with limited scope. Schmitz and MüllerPlathe [25] proposed calculating the total Ps potential in polymers using pair potentials, 

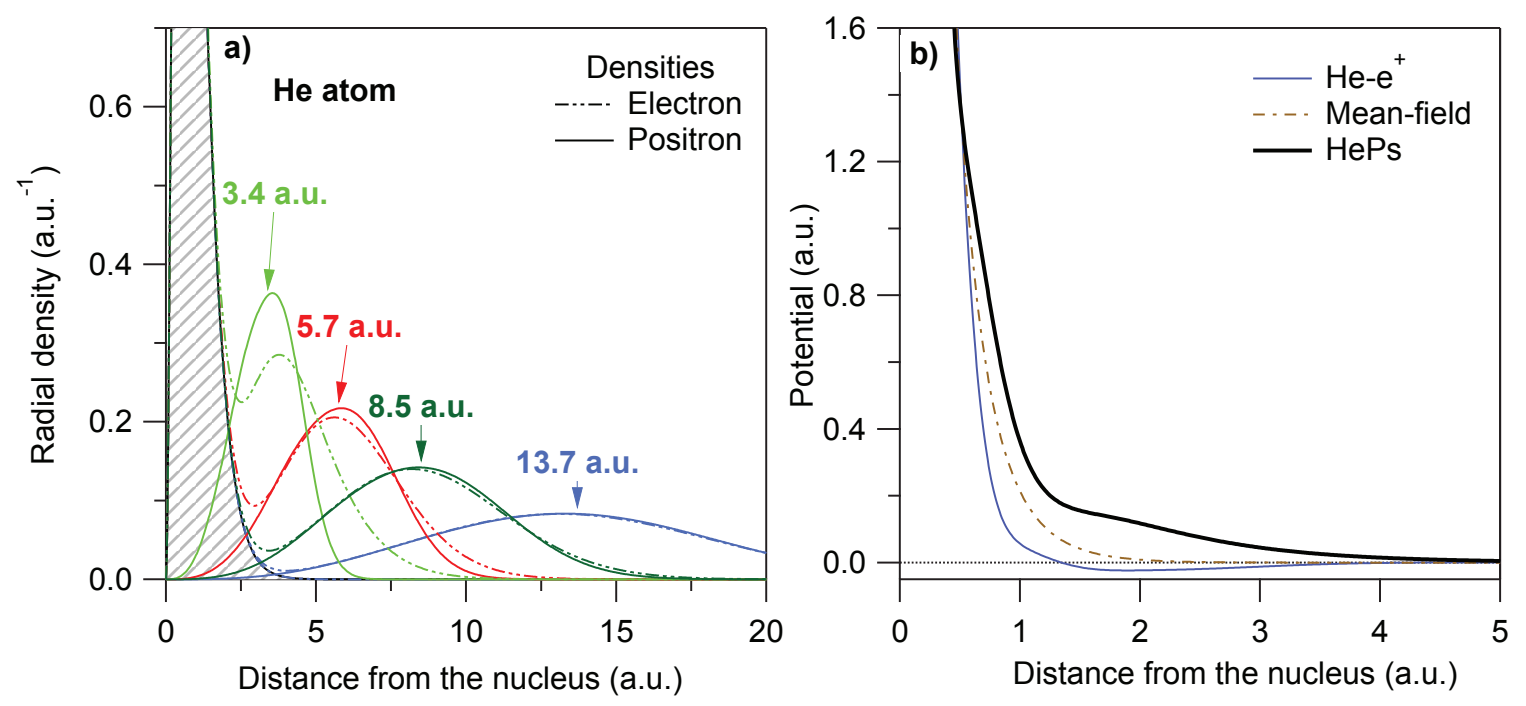

Figure 1. (Color online) Densities and $V_{\text {eff }}$ for HePs systems. (a) Radial distributions of the positron (full lines) and electrons (dashed lines) for four different configurations of the HePs system with $\left\langle r_{p}\right\rangle$ between 3.4 a.u. and 12.1 a.u. The shaded region describes the electron density of a free He atom. Printed with permision from Zubiaga et al . [18]. (b) $\mathrm{V}_{\text {eff }}$ for HePs, positron mean-field Coulomb potential (brown dash-dotted line) and $\mathrm{V}_{\text {eff }}$ for $\mathrm{e}^{+} \mathrm{He}$ (blue line).

and solved the resulting single-particle Schrödinger equation at finite temperature with the path integral Monte-Carlo method. Their pair potentials are parametrized using experimental scattering cross sections and polarizabilities. Saniz et al . [26] used ab-initio electronic structure calculations to define a single particle potential for Ps physisorbed on a surface. Their model could reproduce the binding energy measured on the silica surface.

\subsection{Many-body calculations for the Ps}

The existence of bound states of Ps and small atoms have been explored using accurate many-body ab-initio techniques. The binding energy of the ground state, the annihilation rate and in some cases the densities have been calculated. In many of these studies the many-body wavefunction $\Psi(x)$ has been calculated using the linear combination of $M$ ECG basis components and the stochastic variational methods (SVM) for optimization of $A^{i}$ non-linear coefficient matrices [28].

$\Psi(x)=\sum_{i=1}^{M} c_{i} \mathcal{A}\left[\exp ^{-\frac{1}{2} x A^{i} x}\right] \otimes \chi_{S M s}$,

$A^{i}$ are $N \times N$ coefficient matrices where $N$ is the number of particles, $c_{i}$ are the mixing coefficients of the eigenvectors and $\chi_{S M s}$ is a spin eigenfunction. The number of components $M$ ranges typically between 1000 and 2000. The antisymmetrization operator $\mathcal{A}$ acts on indistinguishable particles and Jacobi coordinate sets $\left\{x_{1}, \ldots, x_{N}\right\}$ with 


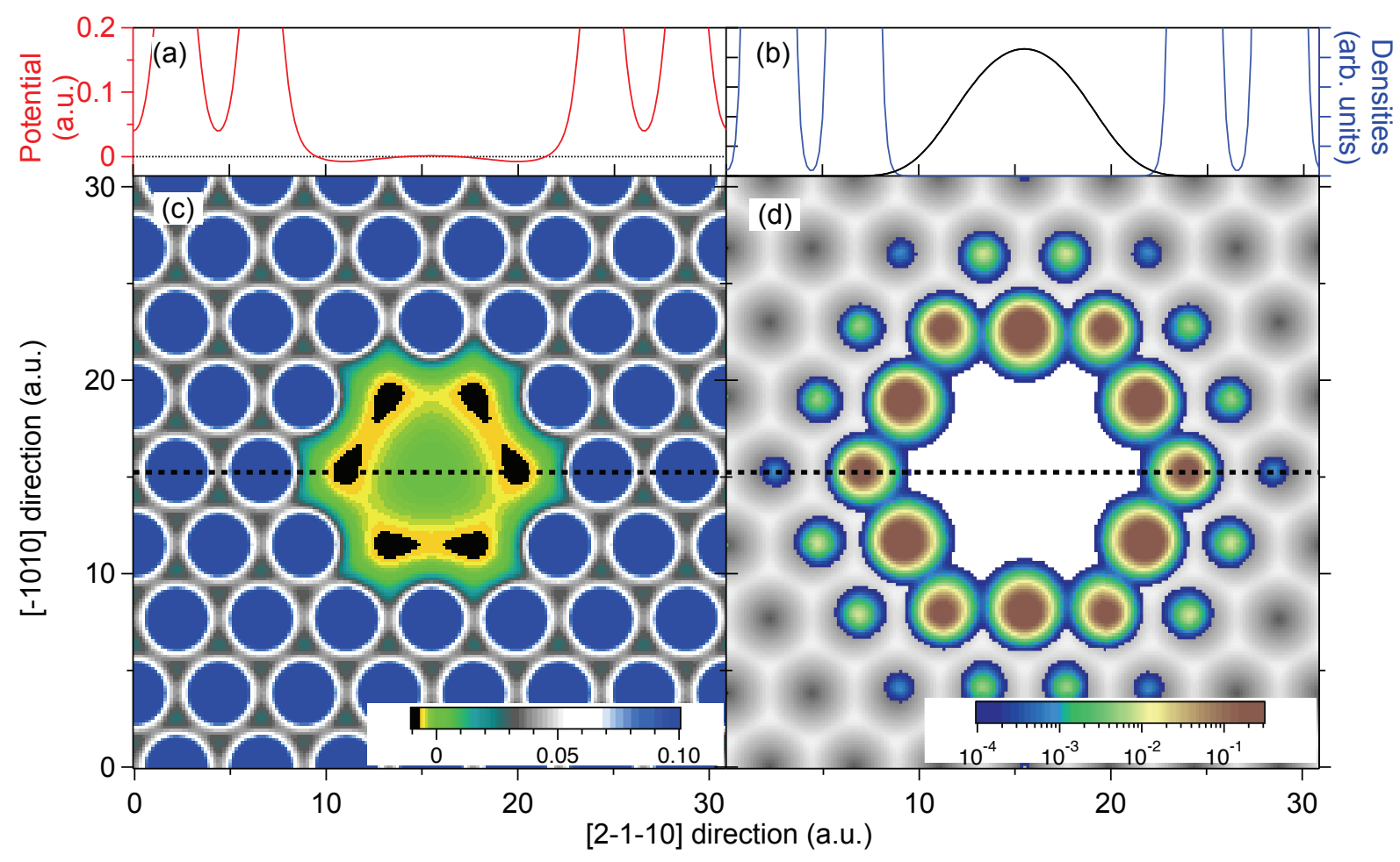

Figure 2. (Color online) Ps inside a spherical void in solid He due to 19 missing atoms (alat $=8.3$ a.u., $r_{s p h}=12.3$ a.u.). Upper panels show (a) the positron potential (red curve), and (b) the positron (black curve) and electron (blue curve) densities plotted along the [2-1-10] direction through the centre of the void. Lower panels show (c) the positron potential (a.u.) and (d) the electron density (gray shade scale) and the local annihilation rate $(1 / \mathrm{ns})$ in the (0001) plane. Reprinted with permision from Zubiaga et al . [27]. Copyright 2015 American Chemical Society.

reduced masses $\mu_{i}=m_{i+1} \sum_{j=1}^{i} m_{j} / \sum_{j=1}^{i+1} m_{j}$ and $\mu_{N}=\sum_{j=1}^{N} m_{j}$ allow a straightforward separation of the centre of mass (CM) movement. The wavefunctions are eigenstates of the non-relativistic Hamiltonian with the kinetic energy of the CM subtracted, i.e.,

$H=\sum_{i} \frac{p_{i}^{2}}{2 m_{i}}-T_{C M}+\sum_{i<j} \frac{q_{i} q_{j}}{r_{i j}}$,

where $\vec{p}_{i}$ is the momentum, $m_{i}$ the mass, and $q_{i}$ the charge of the $\mathrm{i}^{\text {th }}$ particle, $r_{i j}$ is the distance between the $i^{\text {th }}$ and $j^{\text {th }}$ particles and $T_{C M}$ the kinetic energy of the CM.

HPs [29, 30, 31, 32], LiPs [30, 33, 34] and NaPs [30, 34] have been shown to form bound states. Thus far, only a small number of atoms have been considered, but it has been already observed that the atoms able to trap Ps have high electron affinities and open-shell electronic structures. [35] For closed-shell noble gas atoms the electronelectron Pauli repulsion adds to the Coulombic repulsion and the formation of bound states is prevented. Ps interacting with molecules remains to be studied in detail. The electron-electron repulsion will prevent the formation of strongly bound states but the van der Waals attraction may play a role. 
Zubiaga et al . [27, 18, 36] have used ECG-SVM and external confining potentials to study unbound positronic systems including $\mathrm{e}^{+} \mathrm{H}, \mathrm{e}^{+} \mathrm{He}$ and specially HePs. By choosing a large enough confinement radius, the wavefunction could be calculated accurately in the interaction region. The method allowed to obtain accurate interaction energies and positron densities, which were then used to calculate the full-correlation effective potentials for the positron in Ps. The main results and their application to study the pick-off annihilation rate in a molecular soft condensed matter are presented below.

\section{Paving the way to ab-initio positronium simulations in molecular materials}

Our final goal is to describe Ps using quantum-mechanical methods and an atomistic model of the host material. Approximations need to be made having in mind the need to keep the exchange-correlation characteristics and the material specificity of the interaction potential. The similarities between molecules with HOMO-LUMO gaps and closed-shell noble gas atoms in mind, we introduce a model case of Ps interacting with He. In the following, we will describe briefly the calculation of the many-body wavefunction, the construction of an accurate full correlation single-particle potential and the calculation of Ps wavefunction in voids inside solid He.

Figure 1(a) shows the radial distributions of the positron and the electrons in HePs configurations corresponding to several positron mean distance from the nucleus $\left\langle r_{p}\right\rangle$. The electron density of the He atom remains largely undisturbed and the large repulsion felt by the Ps electron and the positron prevent them from entering the electron cloud of the atom. When $\left\langle r_{p}\right\rangle$ is large the density of the Ps electron closely overlaps the density of the positron similarly to Ps in vacuum, whereas for polarized Ps the strong electron-electron repulsion keeps the Ps electron more separated from the nucleus.

\subsection{Construction of single-particle atom-Ps potentials}

We define an atom-positron effective potential $V_{\text {eff }}(r)$ for the Ps-positron by inverting the single-particle Schrödinger equation with the square root of the positron density $n^{+}(r)$ as the eigenfunction and twice the interaction energy $\left(E_{\text {int }}=E_{H e P s}-E_{H e}-E_{P s}\right)$ as the energy eigenvalue, [36]

$V_{e f f}^{P s}(r)=2 E_{i n t}^{P s}+\frac{1}{2} \frac{\nabla^{2} \sqrt{n^{+}(r)}}{\sqrt{n^{+}(r)}}$.

$V_{e f f}^{P s}$ is a Ps positron local interaction potential for low energy elastic scattering when the excitation probability of the target is negligible. It is equivalent to the exact Kohn-Sham potential for a single positron within the two-component DFT [36]. By construction, the asymptotic value of $\mathrm{V}_{\text {eff }}$ is zero far from the nucleus.

$\mathrm{V}_{\text {eff }}$ for HePs, shown in Figure $1(\mathrm{~b})$, remains purely repulsive until 8 a.u. and thereafter it is only marginally attractive. The long-range repulsive tail reflects the 
electron-electron repulsion felt by the electron in Ps and the confinement kinetic energy of the light Ps [37]. $\mathrm{V}_{\text {eff }}$ for HePs and $\mathrm{e}^{+} \mathrm{He}$ are dominated by the mean-field Coulomb positron potential (without the electron-positron correlation) close to the nucleus, $\mathrm{r}<1$ a.u. (figure $1(\mathrm{~b})$ ). However, the $\mathrm{V}_{\text {eff }}$ for $\mathrm{e}^{+} \mathrm{He}$, does not contain exchange repulsion and decays fast to zero [36] following $r^{-4}$.

\subsection{Modeling of Ps in condensed matter}

In soft matter, molecules bind through weak dispersion interactions and their electronic properties are not substantially modified in the condensed state. The superposition of single-particle atom-Ps interaction potentials $\mathrm{V}_{\text {eff }}$ is a good approximation to the total Ps-positron potential in these materials. The positron density and the groundstate energy can then be calculated by solving a single particle Schrödinger equation for the superposition potential. In the present implementation, the problem is discretized on a three-dimensional real-space grid and solved by using a numerical relaxation finite-difference technique and periodic boundary conditions [38, 39]. The electron density is calculated as a superposition of atomic densities obtained from many-body calculations [18]. It is kept frozen when the Schrödinger equation is solved for the Ps positron. The pick-off annihilation rate $\left(\lambda_{\mathrm{c}}^{\mathrm{po}}\right)$ of o-Ps can be calculated to a good approximation from the overlap integral of the positron and matter electron densities [18]

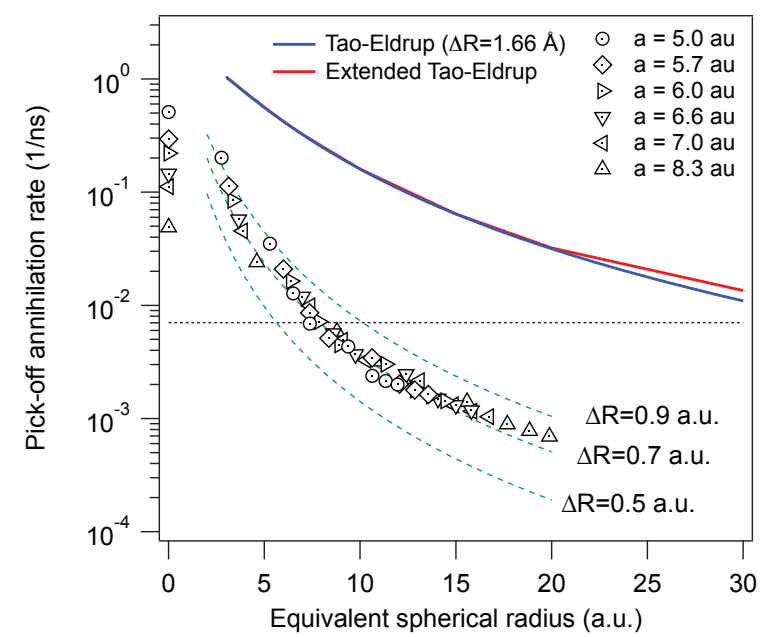

Figure 3. (Color online) Ps pick-off annihilation rates for voids in solid He of different densities (lattice constant $a$ ) plotted against the equivalent spherical radius of the void. The light green dashed lines are calculated using the Tao-Eldrup model with different electron layer widths $\Delta \mathrm{R}$. The dark blue line is the Tao-Eldrup curve for $\Delta \mathrm{R}=3.14$ a.u. $(1.66 \AA)$ commonly used to fit experimental results. The red line is the Extended TaoEldrup model including excited Ps states according to a Boltzmann distribution at room temperature [23]. The horizontal dotted line marks the self-annihilation rate of o-Ps. Reprinted with permision from Zubiaga et al . [27]. Copyright 2015 American Chemical Society. 

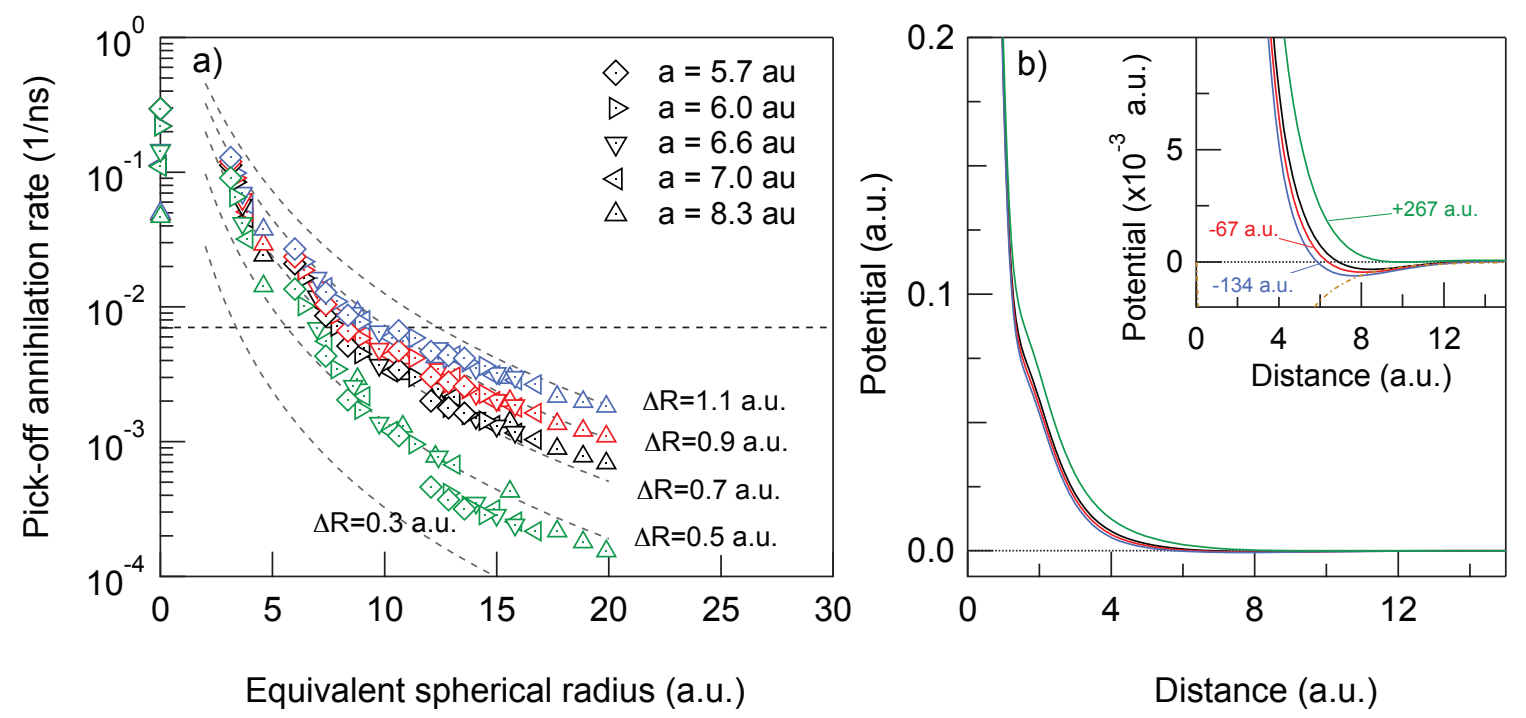

Figure 4. (Color online) Modified van der Waals interaction. (a) Ps pick-off annihilation rates in bulk and inside voids are plotted versus the equivalent spherical radius of the void. The original $\mathrm{V}_{\text {eff }}$ (black markers) and the modified $\mathrm{V}_{\text {eff }}$ with $\Delta \mathrm{C}_{6}$ $=-67$ (red markers), $\Delta \mathrm{C}_{6}=-134$ (blue markers) and $\Delta \mathrm{C}_{6}=+267.4$ (green markers) are shown. The dashed grey lines are obtained from the TE model for spherical voids. The dashed horizontal line marks the self-annihilation rate of o-Ps. (b) The original $\mathrm{V}_{\text {eff }}$ and the potentials with the modified van der Waals term. The inset shows a detail of the potential well. The dash-dotted orange line fits the van der Waals attractive contribution of $\mathrm{V}_{\text {eff }}$ as explained in the text. Reprinted with permision from Zubiaga et al . [27]. Copyright 2015 American Chemical Society.

setting the enhancement factor equal to the unity:

$\lambda_{\mathrm{c}}^{\mathrm{po}}=\pi r_{0}^{2} c \int n_{+}(\mathbf{r}) n_{-}(\mathbf{r}) d \mathbf{r}$,

where $r_{0}$ is the classical electron radius, $c$ is the speed of light, $n_{+}(\mathbf{r})$ is the positron density, and $n_{-}(\mathbf{r})$ is the total electron density of the material.

Below $15 \mathrm{~K}^{4} \mathrm{He}$ crystallizes in a hexagonal close-packed phase when the pressure is above 25 bar [40] and its lattice parameter vary between 8.3 a.u. at 35 bar and 4.0 a.u. at 4.9 kbar. Rytsölä et al . [41] measured pick-off annihilation rates ranging between $0.0121 / \mathrm{ns}$ (25 bar) and $0.0181 / \mathrm{ns}$ (63 bar).

\subsection{Quasi-spherical voids in solid He}

The pick-off annihilation rate in solid He can be calculated within the scheme introduced above. The Ps-positron density is calculated in six supercells comprising 448 He atoms, each with lattice parameters $\left(\mathrm{a}_{\mathrm{lat}}\right.$ ) between 5.0 a.u and 8.3 a.u. In each lattice, quasispherical voids with open volumes ranging from 1 to 81 missing He atoms have been introduced. By taking a large enough supercell we ensure that the positron density is low enough at the supercell boundary so that the interaction between periodic images is negligible and the total energy of the positron is well converged 
The long tail of $\mathrm{V}_{\text {eff }}$ increases the total potential in the bulk and only inside a void the total potential is low enough to confine Ps (Figure 2(a) and 2(c)). The weak dispersion attraction of He induces a shallow potential well near the wall of the void. The main contribution to the pick-off annihilation rate in Figure 2(d) comes from the atoms forming the wall of the void. The second neighbours also contribute to smaller extent, specially when the void is small.

The pick-off annihilation rates, $\lambda_{\mathrm{c}}^{\mathrm{po}}$, in Figure 3 show how the pick-off annihilation rate decreases as the size of the void increases $\left(r_{s p h} \geq 3\right.$ a.u.). Moreover, the decay rate does not depend on the lattice parameter. The interaction energy follows also a common curve for voids of the same size (not shown, see reference [27]). The $\lambda_{\mathrm{c}}^{\text {po }}$ in solid He is very low compared to the values of the Tao-Eldrup model $\lambda_{T E}^{p o}$ because of the low polarizability of He. However, $\lambda_{\mathrm{c}}^{\text {po }}$ can be fitted by the Tao model with $\Delta \mathrm{R}=0.7$ a.u. for equivalent spherical radii larger than 6 a.u.

The pick-off annihilation rates measured by Rytsöla et al . [41] at $1.4 \mathrm{~K}$ correspond to void radii ranging from 5.7 a.u. at 63 bar to 6.5 a.u. at 25 bar. At 25 bar He transforms into the liquid phase and the bubble radius increases to 6.8 a.u. At $4.2 \mathrm{~K}$ the bubble radius of liquid He ranges between 5.9 a.u. and 6.9 a.u. In polymers, the Tao-Eldrup model with $\Delta \mathrm{R}=1.66$-1.8 a.u. yields radii ranging from 3.5 a.u. to 8 a.u. In contrast, a spherical finite potential well yield values between 12 a.u. and 27 a.u. [41]. In organic liquids the values range from 7 a.u. to 11 a.u. [3].

\subsection{Dispersion interaction}

Even relatively weak dispersion attractions can have an impact in the pick-off annihilation of Ps confined inside open volume pockets or voids. This is so because the contact density of the Ps-positron and the tail of the electron density is small. The

effect has been estimated within the present model by adding to $V_{\text {eff }}$ a van der Waals term $\mathrm{V}(\mathrm{R})=\mathrm{g}(\alpha \mathrm{R}) \Delta \mathrm{C}_{6} / \mathrm{R}^{6}$. Here, the damping function $g(\alpha R)$ prevents unphysical behaviour of $\mathrm{V}(\mathrm{R})$ as $\mathrm{Ps}$ approaches the electron cloud of He. [42]

The strengthened van der Waals interaction does not affect the pick-off annihilation rate in small voids but for voids larger than 8-9 a.u. it remains larger than for the original $\mathrm{V}_{\text {eff }}$. The pick-off annihilation rates deviate from the Tao model and tend to saturate at values which depend on the intensity of the van der Waals term. For the strongest dispersion considered the electron-layer thickness $\Delta R$ that best fits the data ranges from 0.7 a.u. for voids of $r_{s p h} \sim 5$ a.u. to 1.1 a.u. when $r_{s p h} \sim 20$ a.u. On the other hand, the annihilation rates for the modified $V_{\text {eff }}$ without any dispersion attraction are well described by the TE model for voids larger than 8 a.u. with $\Delta \mathrm{R}=0.4-0.5$ a.u. This difference is caused by a resonant increase of the positron density near the walls of the void correlated with the depth of the attractive well. 


\section{Two-particle picture of interacting electron-positron systems}

While the models discussed above use results from many-body quantum calculations to define practical effective potentials aimed at describing Ps in soft matter and the associated annihilation parameters, it is desirable to search for a more fundamental and general description of correlated positron-electron systems that can involve both free positrons, and most importantly, formation of correlated states such as Ps. For that purpose, let us suppose now that we know the exact many-body ground state of our system and want to somehow analyze and quantify the nature of correlated electron-positron states (e.g. Ps-like) present. The properties of interacting manyparticle systems in their ground state are determined by the time-independent manybody Schrödinger equation and its ground-state solution, the many-body wave function, $\Psi\left(\mathbf{r}_{1}, \mathbf{r}_{2}, \ldots, \mathbf{r}_{N}\right)$, a multi-dimensional quantity which is difficult to solve and represent in a general case. A simpler quantity providing access to expectation values of one and two-body operators is the two-body reduced density matrix (2-RDM). The 2-RDM of a many-body ground state can be obtained by summing over, or contracting, all

particles except two. As an example, the zero-temperature electron-positron 2-RDM can be written as

$\Gamma^{e p}\left(\mathbf{r}_{p}, \mathbf{r}_{e} ; \mathbf{r}_{p}^{\prime}, \mathbf{r}_{e}^{\prime}\right)=N_{e} N_{p} \int d \mathbf{r}_{3} \ldots d \mathbf{r}_{N} \Psi\left(\mathbf{r}_{p}, \mathbf{r}_{e}, \mathbf{r}_{3}, \ldots, \mathbf{r}_{N}\right) \Psi^{*}\left(\mathbf{r}_{p}^{\prime}, \mathbf{r}_{e}^{\prime}, \mathbf{r}_{3}, \ldots, \mathbf{r}_{N}\right)$,

where $N_{e}$ and $N_{p}$ are the numbers of electrons and positrons, $\mathbf{r}_{i}$ is the position of particle $i$, and the subscripts $e$ and $p$ denote electrons and positrons with fixed spin directions. The spin indices could be carried in the equations together with the coordinates, but we fix the spins in the numerical examples below, and thus adopt such a convention in our formulae. Here, we assume that the first argument of the properly antisymmetrized wave function $\Psi\left(\mathbf{r}_{1}, \mathbf{r}_{2}, \ldots, \mathbf{r}_{N}\right)$ is the positron coordinate, whereas the rest of the coordinates are for electrons.

The electron-positron 2-RDM is the quantity of interest when one is interested in the physical properties of positronium formation and positron annihilation, such as the annihilation rate or the momentum density of annihilating pairs. In principle, any expectation value of a one or two-body observable can be evaluated using the 2-RDM, such that it contains all the relevant information about individual particles and particle pairs related to the ground state wave function. However, it is not straightforward to solve the 2-RDM without first solving the full many-particle ground state, since candidate 2-RDMs have to fulfill the so-called $N$-representability conditions [43, 44] that guarantee the 2-RDMs to correspond to true wave functions. There has been much work done about the theory and numerical methods as well to use RDMs instead of the full wave function to describe the state of a physical system [43, 44, 45, 46, 47]. Another rigorous avenue to obtain amplitudes of physical processes, such as positron scattering and annihilation, is to use perturbative expansions and diagrammatic many-body theory [48, 49, 50]. 
When analyzing correlated systems with electrons and positrons, it is useful to find a description involving interacting pairs of electrons and positrons. Instead of isolating just a single pair of distinguishable particles that are chosen to represent Ps, the proper antisymmetrization can be taken into account by using the 2-RDM. It can be expanded by two-particle states, or geminals $\left\{\omega_{i}\right\}$ as

$\Gamma^{e p}\left(\mathbf{r}_{p}, \mathbf{r}_{e} ; \mathbf{r}_{p}^{\prime}, \mathbf{r}_{e}^{\prime}\right)=\sum_{i j} b_{i j} \omega_{i}\left(\mathbf{r}_{p}, \mathbf{r}_{e}\right) \omega_{j}^{*}\left(\mathbf{r}_{p}^{\prime}, \mathbf{r}_{e}^{\prime}\right)$.

Furthermore, the 2-RDM is Hermitian with respect to the first and second pairs of coordinates or indices, and it can be therefore diagonalized as

$\int d \mathbf{r}_{p}^{\prime} d \mathbf{r}_{e}^{\prime} \Gamma^{e p}\left(\mathbf{r}_{p}, \mathbf{r}_{e} ; \mathbf{r}_{p}^{\prime}, \mathbf{r}_{e}^{\prime}\right) \alpha_{j}\left(\mathbf{r}_{p}^{\prime}, \mathbf{r}_{e}^{\prime}\right)=g_{j} \alpha_{j}\left(\mathbf{r}_{p}, \mathbf{r}_{e}\right)$

The resulting orthonormal two-particle eigenfunctions $\left\{\alpha_{j}\right\}$ are called natural geminals, and the eigenvalues $g_{j}$ are the real, non-negative weights corresponding to classical (non-interfering) occupations. Most importantly, the 2-RDM expansion is diagonal in the basis of the natural geminals

$\Gamma^{e p}\left(\mathbf{r}_{p}, \mathbf{r}_{e} ; \mathbf{r}_{p}^{\prime}, \mathbf{r}_{e}^{\prime}\right)=\sum_{j} g_{j} \alpha_{j}\left(\mathbf{r}_{p}, \mathbf{r}_{e}\right) \alpha_{j}^{*}\left(\mathbf{r}_{p}^{\prime}, \mathbf{r}_{e}^{\prime}\right)$.

By tracing both sides, it follows that $\sum_{j} g_{j}=N_{p} N_{e}$.

In practice, the 2-RDM elements can be numerically evaluated by expanding in suitable single-particle orbital bases, say $\left\{\phi_{i}^{e}\right\}$ and $\left\{\phi_{i}^{p}\right\}$, such that

$\Gamma^{e p}\left(\mathbf{r}_{p}, \mathbf{r}_{e} ; \mathbf{r}_{p}^{\prime}, \mathbf{r}_{e}^{\prime}\right)=\sum_{i j k l} \rho_{i j k l}^{e p} \phi_{i}^{p *}\left(\mathbf{r}_{\mathbf{p}}^{\prime}\right) \phi_{j}^{e *}\left(\mathbf{r}_{\mathbf{e}}^{\prime}\right) \phi_{k}^{p}\left(\mathbf{r}_{\mathbf{p}}\right) \phi_{l}^{e}\left(\mathbf{r}_{\mathbf{e}}\right)$

where the factors are $\rho_{i j k l}^{e p}=\left\langle\hat{a}_{i p}^{\dagger} \hat{a}_{j e}^{\dagger} \hat{a}_{l e} \hat{a}_{k p}\right\rangle$. Such a four-index sum is less elegant than Eq. (10) to analyze correlations, and it is more difficult to develop accurate approximations for such an expression. Typically a good choice for the single-particle basis is the set of natural orbitals $\left\{\varphi_{j}\right\}$ that diagonalize the 1-RDM, written for instance for the electron as

$\gamma^{e}\left(\mathbf{r}_{e}, \mathbf{r}_{e}^{\prime}\right)=N_{e} \int d \mathbf{r}_{p} d \mathbf{r}_{3} \ldots d \mathbf{r}_{N} \Psi\left(\mathbf{r}_{p}, \mathbf{r}_{e}, \mathbf{r}_{3}, \ldots, \mathbf{r}_{N}\right) \Psi^{*}\left(\mathbf{r}_{p}, \mathbf{r}_{e}^{\prime}, \mathbf{r}_{3}, \ldots, \mathbf{r}_{N}\right)$,

such that

$\gamma^{e}\left(\mathbf{r}_{e}, \mathbf{r}_{e}^{\prime}\right)=\sum_{j} n_{j}^{e} \varphi_{j}\left(\mathbf{r}_{e}\right) \varphi_{j}^{*}\left(\mathbf{r}_{e}^{\prime}\right)$,

where $n_{j}^{e}$ are the eigenvalues, namely the occupations of the natural orbitals.

The 2-RDM can be understood intuitively as a mixed state of two particles, which can be written as a sum of pure states with certain classical probabilities. The observable averages can also be written in a convenient way, namely as a sum of the observable averages of the pure state natural geminals weighted by the probabilities $g_{j}$. For instance, the interaction energy between positrons and electrons (one spin component only) is written in a compact form as

$$
\begin{aligned}
E^{e p}=-\frac{1}{2} \int d \mathbf{r}_{p} d \mathbf{r}_{e} \frac{\Gamma^{e p}\left(\mathbf{r}_{p}, \mathbf{r}_{e} ; \mathbf{r}_{p}, \mathbf{r}_{e}\right)}{\left|\mathbf{r}_{p}-\mathbf{r}_{e}\right|} \\
=\sum_{j} g_{j}\left(-\frac{1}{2} \int d \mathbf{r}_{p} d \mathbf{r}_{e} \frac{\left|\alpha_{j}\left(\mathbf{r}_{p}, \mathbf{r}_{e}\right)\right|^{2}}{\left|\mathbf{r}_{p}-\mathbf{r}_{e}\right|}\right) .
\end{aligned}
$$


Similarly, the contact density (density of electrons at the positron's site) can be written using the natural geminals as

$$
\left\langle\hat{n}_{-}(\mathbf{r}) \hat{n}_{+}(\mathbf{r})\right\rangle=\Gamma^{e p}(\mathbf{r}, \mathbf{r} ; \mathbf{r}, \mathbf{r})=\sum_{j} g_{j}\left|\alpha_{j}(\mathbf{r}, \mathbf{r})\right|^{2},
$$

where $\hat{n}_{-}(\mathbf{r})$ and $\hat{n}_{+}(\mathbf{r})$ are the operators for electron and positron densities, respectively. The contact density provides the positron annihilation rate

$\lambda \propto \int d \mathbf{r} \Gamma^{e p}(\mathbf{r}, \mathbf{r} ; \mathbf{r}, \mathbf{r})=\sum_{j} g_{j} \int d \mathbf{r}\left|\alpha_{j}(\mathbf{r}, \mathbf{r})\right|^{2}$.

Here, the spin parts are not explicitly included, and a projection to the singlet spin state is required to evaluate the $2 \gamma$ annihilation rate. Furthermore, if electrons and positrons are approximated as independent, the contact density becomes

$\left\langle\hat{n}_{-}(\mathbf{r}) \hat{n}_{+}(\mathbf{r})\right\rangle \approx\left\langle\hat{n}_{-}(\mathbf{r})\right\rangle\left\langle\hat{n}_{+}(\mathbf{r})\right\rangle=n_{-}(\mathbf{r}) n_{+}(\mathbf{r})$.

While contact density is strictly a two-body observable, the independent particle approximation (IPA) reduces it to the single particle picture. The true and IPA contact densities can be related by defining the usual enhancement factor $\gamma(\mathbf{r})$ for particular inhomogeneous model systems, as $\Gamma^{e p}(\mathbf{r}, \mathbf{r} ; \mathbf{r}, \mathbf{r})=\gamma(\mathbf{r}) n_{-}(\mathbf{r}) n_{+}(\mathbf{r})$. The enhancement factor describes the short range screening of the positron by the electrons, which increases the annihilation rate compared to the IPA. This information is not contained in the average one-body densities $n_{-}(\mathbf{r})$ and $n_{+}(\mathbf{r})$, which are the fundamental quantities in two-component density-functional calculations.

The momentum density of annihilating electron-positron pairs can be written in a simple and intuitive form using the natural geminals as [51]

$\rho(\mathbf{p}) \propto \int d \mathbf{r} d \mathbf{r}^{\prime} e^{-i \mathbf{p} \cdot\left(\mathbf{r}-\mathbf{r}^{\prime}\right)} \Gamma^{e p}\left(\mathbf{r}, \mathbf{r} ; \mathbf{r}^{\prime}, \mathbf{r}^{\prime}\right)=\sum_{j} g_{j}\left|\int d \mathbf{r} e^{-i \mathbf{p} \cdot \mathbf{r}} \alpha_{j}(\mathbf{r}, \mathbf{r})\right|^{2}$,

where again the spin state has to be projected to the singlet state, since the formula is exclusively for $2 \gamma$ annihilation. That is, the momentum density is the squared amplitude of the Fourier transformed electron-positron wave function with coinciding coordinates. The contact density can be written in the IPA by using $\Gamma^{e p}\left(\mathbf{r}, \mathbf{r} ; \mathbf{r}^{\prime}, \mathbf{r}^{\prime}\right) \approx \gamma^{e}\left(\mathbf{r}, \mathbf{r}^{\prime}\right) \gamma^{p}\left(\mathbf{r}, \mathbf{r}^{\prime}\right)$, and then plugging in for instance the natural orbital expansion Eq. (13), resulting in

$\int d \mathbf{r} d \mathbf{r}^{\prime} e^{-i \mathbf{p} \cdot\left(\mathbf{r}-\mathbf{r}^{\prime}\right)} \Gamma^{e p}\left(\mathbf{r}, \mathbf{r} ; \mathbf{r}^{\prime}, \mathbf{r}^{\prime}\right) \approx \sum_{j k} n_{j}^{e} n_{k}^{p}\left|\int d \mathbf{r} e^{-i \mathbf{p} \cdot \mathbf{r}} \varphi_{j}^{e}(\mathbf{r}) \varphi_{k}^{p}(\mathbf{r})\right|^{2}$

where, more often than not, the occupations $n_{j}^{e}$ and $n_{k}^{p}$ take integer values ( 0 or 1$)$ such as in mean-field or conventional DFT calculations, or in any method that is inherently in the single particle picture.

It is possible to develop the enhancement models further. By comparing for instance Eq. (15) to Eq. (17), or Eq. (18) to Eq. (19), state-dependent enhancement factors can be defined that relate natural geminals to the product states of natural orbitals (the limit in the absence of electron-positron interaction) as [51]

$\alpha_{j}(\mathbf{r}, \mathbf{r})=\sqrt{\gamma_{j}(\mathbf{r})} \varphi_{0}^{p}(\mathbf{r}) \varphi_{j}^{e}(\mathbf{r})$ 

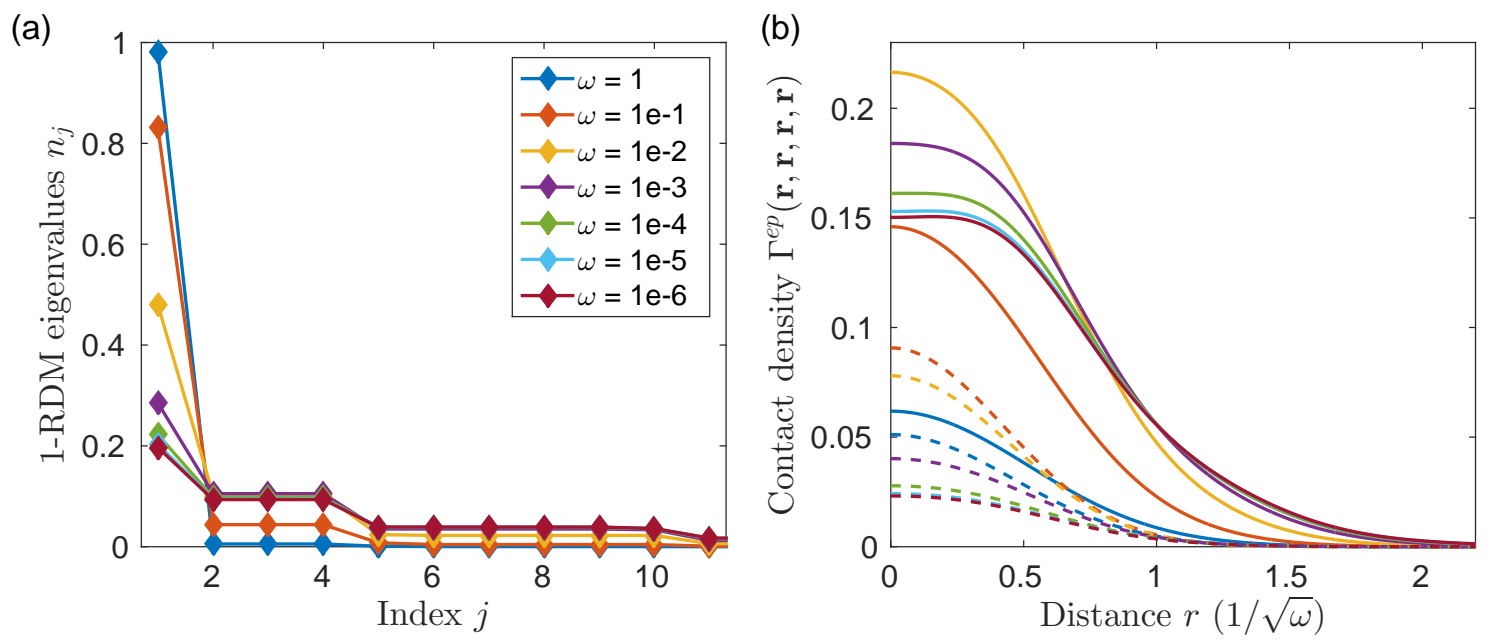

Figure 5. (a) Electron or positron 1-RDM eigenvalues (in descending order) of isolated Ps ground state in various isotropic harmonic confinements $\omega$. (b) Contact density $\Gamma^{e p}(\mathbf{r}, \mathbf{r}, \mathbf{r}, \mathbf{r})$ as a function of the distance from the harmonic trap center (solid lines), and contact density using the independent particle approximation of Eq. (17) (dashed lines).

where $\varphi_{0}^{p}$ is the positron state, and $\left\{\varphi_{j}^{e}\right\}$ are the electron natural orbitals. Such an enhancement model works reasonably well, if the positron natural occupations are described by a step function, since in principle all the partially occupied states have to be included as well. It is crucial that only those natural geminals and natural orbitals with the same shell or symmetry properties are related, or otherwise the enhancement factors would not only describe the enhancement of the annihilation rate but also how the geminal and orbital product symmetries differ. A straightforward method could be to continuously mapping the natural geminals to the natural orbital product states by gradually tuning the system parameters by increasing the correlations in the system. Because of this and since the natural geminals and their eigenvalues in Eq. (18) are unique, the expressions of Eq. (10) and Eq. (20) can be, in principle, used to determine practical enhancement models beyond the IPA to be used in the context of large-scale electronic-structure or band-structure calculations [51]. Other similar statedependent enhancement factors have been defined and evaluated in Refs. [49, 50], where a diagrammatic many-body approach is used to solve the positron annihilation on noble gas atoms.

\subsection{Isolated positronium}

We performed numerical simulations of a model system containing a positron and several electrons in an isotropic harmonic trap, in order to illustrate the one-body and twobody pictures of natural orbitals and natural geminals, respectively. The interacting 
$N$-particle Hamiltonian is written as

$\hat{H}=\sum_{i=1}^{N}\left(\frac{1}{2} \hat{\mathbf{p}}_{i}^{2}+\frac{1}{2} \omega^{2} \hat{\mathbf{r}}_{i}^{2}\right)+\sum_{i<j} \frac{q_{i} q_{j}}{\left|\hat{\mathbf{r}}_{i}-\hat{\mathbf{r}}_{j}\right|}$,

where $\omega$ is the harmonic confinement strength and $q_{i}$ is the charge of particle with index $i$. We solved the ground state manifold using exact diagonalization with a basis cutoff procedure. Namely, only the lowest 8 non-interacting energy shells (120 orbitals) of the non-interacting problem were included to construct the many-body basis configurations. In three dimensions, the non-interacting energy shells have $1,3,6,10, \ldots$ orbitals. By lowering the confinement $\omega$, we can tune the interaction with respect to the energy gaps between the energy shells, effectively approaching the limit of no external potential, yet trapping the particles due to small $\omega$ and the cutoff of orbitals.

In the case of an isolated Ps, namely a system containing a positron and an electron, the 2-RDM of the ground state is the full density matrix of the pure ground state $\Psi\left(\mathbf{r}_{p}, \mathbf{r}_{e}\right)$. The natural geminal expansion is written trivially as

$\Gamma^{e p}\left(\mathbf{r}_{p}, \mathbf{r}_{e} ; \mathbf{r}_{p}^{\prime}, \mathbf{r}_{e}^{\prime}\right)=\Psi\left(\mathbf{r}_{p}, \mathbf{r}_{e}\right) \Psi^{*}\left(\mathbf{r}_{p}^{\prime}, \mathbf{r}_{e}^{\prime}\right)$

where now only the geminal corresponding to the Ps wave function is occupied.

We hoped to catch Ps-like bound states proportional to $\exp \left(-\left|\mathbf{r}_{p}-\mathbf{r}_{e}\right| / a\right)$, such that the state had been additionally localized due to the confinement and orbital cutoff. It is evident that such a state with interparticle distance in the exponent cannot be written as a single product of uncorrelated orbitals, namely as a separable state. As a result, at least some of the 1-RDM eigenvalues must have values other than 0 (fully unoccupied) and 1 (fully occupied).

We evaluated the 1-RDM eigenvalues of the isolated Ps ground state, and they are shown in Fig. 5(a). With a high value of the confinement $\omega$, the closed shell system is well approximated by the product state of the most occupied electron and positron natural orbitals, namely $\varphi_{0}^{p}\left(\mathbf{r}_{p}\right) \varphi_{0}^{e}\left(\mathbf{r}_{e}\right)$, since the confinement is too strong to let particles occupy the other energy shells. However with low $\omega$, the single-particle picture breaks down in the sense that some of the 1-RDM eigenvalues take values between 0 and 1 . Such partial occupations indicate correlations and entanglements with respect to the given partitioning, in this case between the positron and electron. Furthermore, the shell structure is clearly visible in the 1-RDM eigenvalues, and with low enough $\omega$, the total occupation in the second lowest (p-type) energy shell is already higher than in the lowest (s-type) energy shell.

To further illustrate the correlations present in the system, the electron-positron contact density is compared to the contact density in the independent particle approximation in Fig. 5(b). With lower $\omega$ also corresponding to stronger correlations, the tail of the contact density is longer. On the other hand, the contact density close to the potential center seems to saturate, as the system is expected to form Ps-like bound states. The independent particle approximation gives systematically too low contact density values. It works moderately for the $\omega=1$ case, and poorly for the other cases. Despite the enhancement factor can be neglected in few cases as the pick-off annihilation 

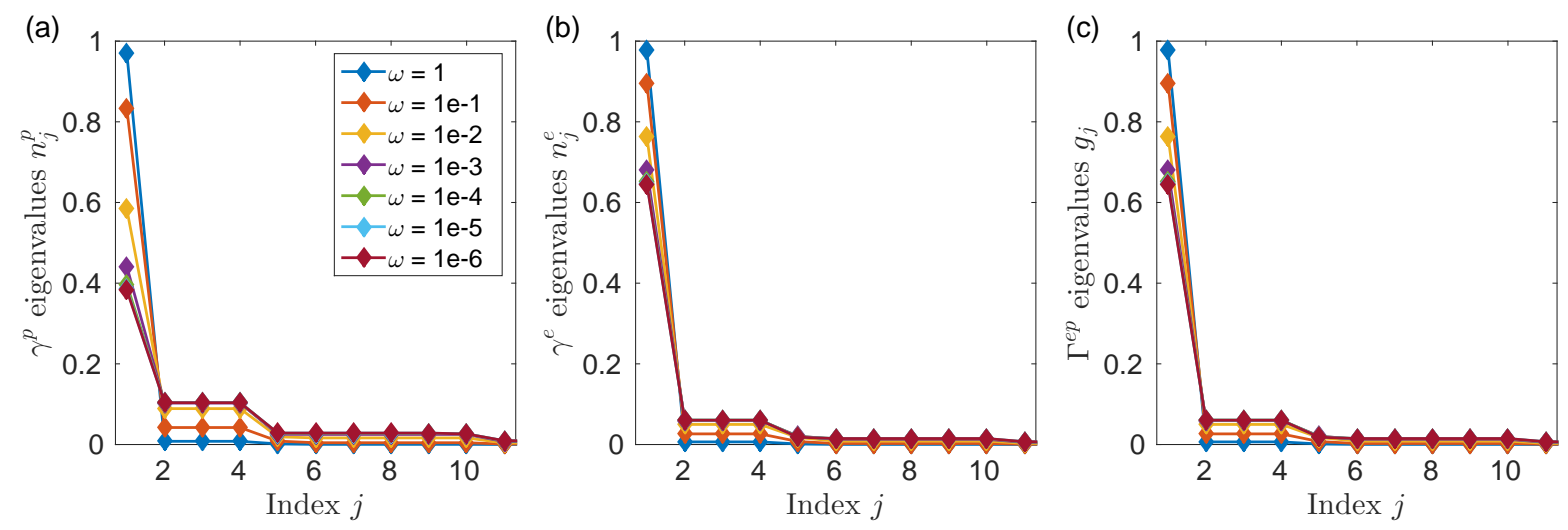

Figure 6. (a) Positron 1-RDM $\gamma^{p}$ eigenvalues $n_{j}^{p}$ (in descending order), (b) electron 1-RDM $\gamma^{e}$ eigenvalues $n_{j}^{e}$ (in descending order), and (c) electron-positron 2-RDM $\Gamma^{e p}$ eigenvalues $g_{j}$ (in descending order) of $\mathrm{Ps}^{-}$ground state, namely a positron and two electrons of opposite spins, in various harmonic confinement strengths $\omega$.

of o-Ps discussed above, a general approach needs enhancement models within the single particle picturetodescribe many-bodyquantities such as contact densities, annihilation rates andmomentum densities of annihilating electron-positron pairs.

\subsection{Negative positronium}

It is interesting to consider the case of negative positronium, $\mathrm{Ps}^{-}$, that has one positron and two electrons of opposite spins. The natural question to ask is then whether the electron-positron pairs within the system form Ps-like bound states, and how the natural geminals would display this. The 1-RDM eigenvalues and electron-positron 2-RDM eigenvalues of the ground state are shown in Fig. 6. The single- and two-particle pictures break down with low enough confinement strength $\omega$, in the sense that the natural occupations are not described by a step function. However, since there are only three particles, the natural orbitals and natural geminals are dual descriptions. Namely, when partitioning a system into two subsets in general, one with $p$ and the other with the remaining $(N-p)$ particles, the corresponding $p$-RDM and $(N-p)$-RDM have the same eigenvalues, and the natural states are related [43]. In fact, the numerical eigenvalues in Fig. 6 of $\Gamma^{e p}$ and $\gamma^{e}$ (or $\Gamma^{e e}$ and $\gamma^{p}$ ) are the same. Furthermore, the ground state, its natural orbitals and natural geminals are related by

$c_{i} \alpha_{i}^{p e}\left(\mathbf{r}_{p}, \mathbf{r}_{e}\right)=\int d \mathbf{r} \varphi_{i}^{e *}(\mathbf{r}) \Psi\left(\mathbf{r}_{p}, \mathbf{r}_{e}, \mathbf{r}\right)$,

where $\left|c_{i}\right|^{2}=g_{i}^{p e}=n_{i}^{e}$. Similarly, the natural orbitals can be obtained by contracting the corresponding natural geminal from the ground state.

In Fig. 6, the highest positron 1-RDM natural occupation in each case is clearly lower than the corresponding highest electron 1-RDM natural occupation. This can be interpreted as the electron pairing with a positron more likely than with another electron, as the positron is more correlated than an electron with respect to the rest of 

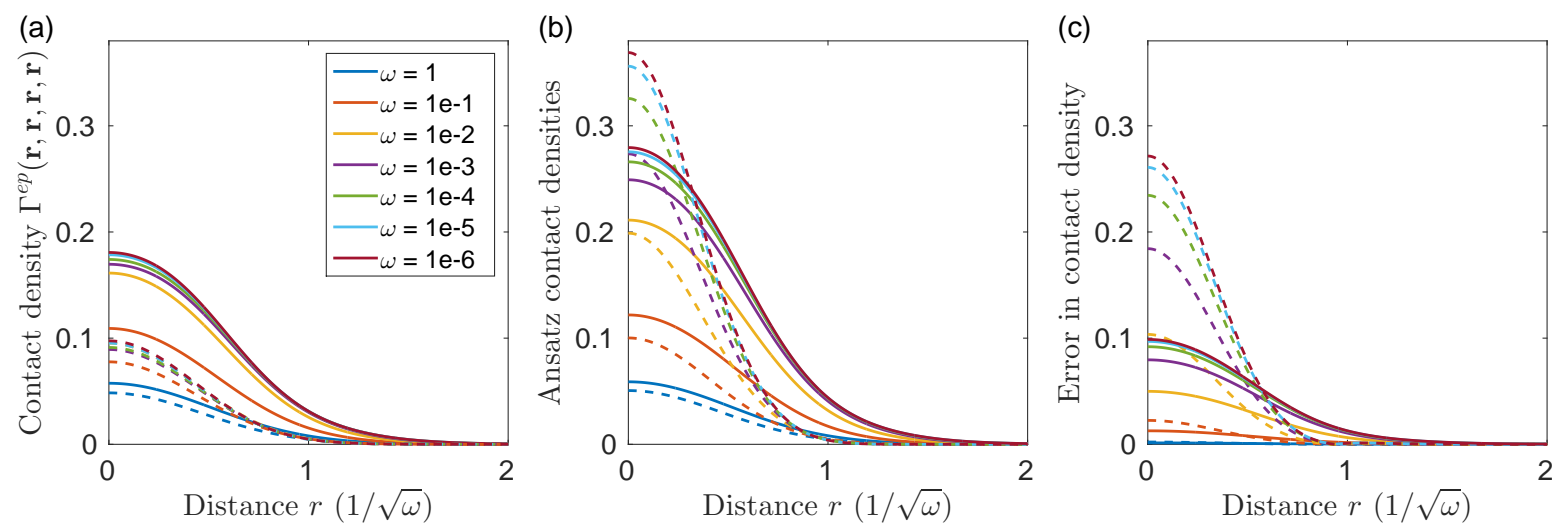

Figure 7. (a) Contact density $\Gamma^{e p}(\mathbf{r}, \mathbf{r}, \mathbf{r}, \mathbf{r})$ of $\mathrm{Ps}^{-}$numerical ground state in various harmonic confinement strengths $\omega$ (solid lines), and the contact densities if using the the independent particle approximation (dashed lines). (b) Contact densities of the first/most occupied natural geminal $\alpha_{0}$ (solid lines), and contact densities of the product state consisting of the first/most occupied natural orbitals of the electron and the positron, namely $\varphi_{0}^{p} \varphi_{0}^{e}$ (dashed lines). (c) The (absolute deviation) errors of the ansätze $\alpha_{0}$ and $\varphi_{0}^{p} \varphi_{0}^{e}$ to the contact density of the numerical ground state.

the system, namely the remaining two particles. The $\mathrm{Ps}^{-}$ground state wave function can be written using the natural states as [43]

$\Psi\left(\mathbf{r}_{p}, \mathbf{r}_{e \uparrow}, \mathbf{r}_{e \downarrow}\right)=\sum_{i} c_{i} \alpha_{i}^{p e}\left(\mathbf{r}_{p}, \mathbf{r}_{e \uparrow}\right) \varphi_{i}^{e}\left(\mathbf{r}_{e \downarrow}\right)$.

The ground state could also be written by partitioning the electrons together, but in that case the linear combination would contain more terms with amplitudes that are still relevant, as indicated by the broader eigenvalue distribution of the positron 1-RDM compared to the electron 1-RDM. Therefore the ground state can be better approximated as a product of an electron orbital and an electron-positron pair wave function.

The contact densities in the $\mathrm{Ps}^{-}$systems with various confiments $\omega$ are shown in Fig. 7(a). They are similar to the Ps cases studied above, but the $\mathrm{Ps}^{-}$contact densities seem to converge from below as $\omega$ is decreased. Furthermore, the tail is slightly shorter in the case of $\mathrm{Ps}^{-}$, indicating that the probability of finding both the positron and an electron at the same position $\mathbf{r}$ further from the trap center is lowered due to the third electron. However, in the highly correlated limit, the contact density at the trap center is slightly increased. Again, the independent particle approximation (dashed lines) systematically results in too low contact density values.

The contact densities and other observable averages can be approximated for instance by assuming only a single geminal or a single product state of orbitals to describe the electron-positron pair. To estimate how such approximations would work, the contact densities of the lowest natural geminal $\alpha_{0}$ and the product state of the lowest natural orbitals $\varphi_{0}^{e} \varphi_{0}^{p}$ are shown in Fig. 7(b). Such ansätze clearly result in too high values of contact densities especially at small distances $r$ from the trap center, due to oversimplification of the natural occupation distributions and overemphasis on 

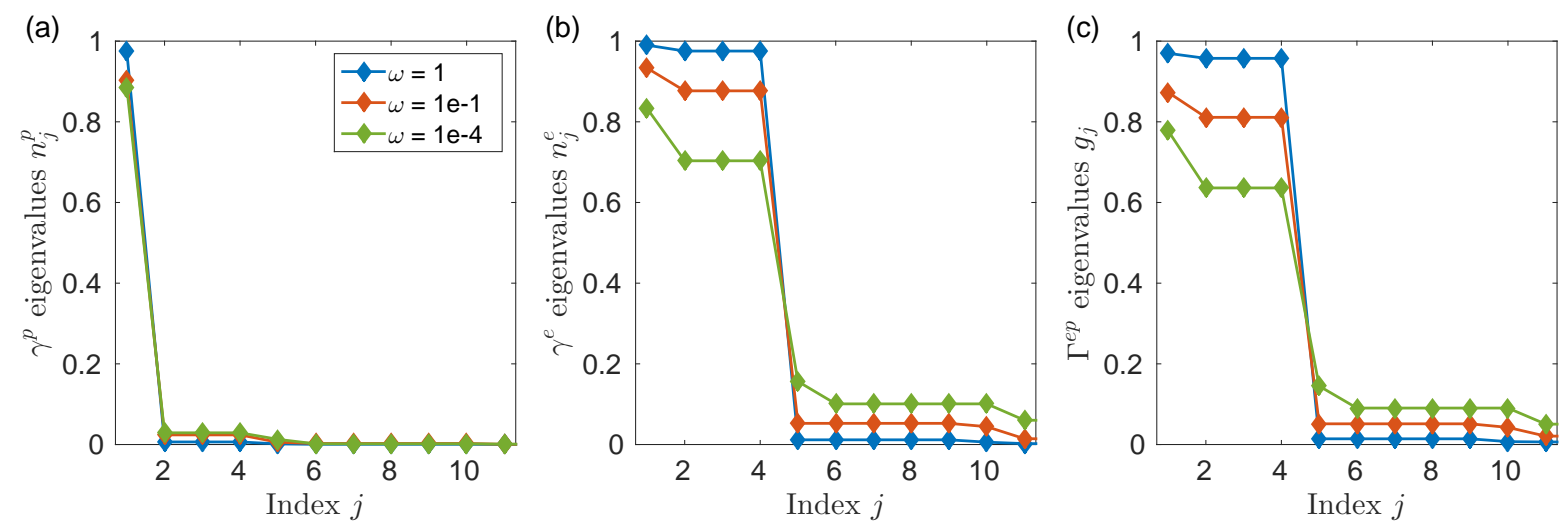

Figure 8. (a) Positron 1-RDM $\gamma^{p}$ eigenvalues $n_{j}^{p}$ (in descending order), (b) electron 1-RDM $\gamma^{e}$ eigenvalues $n_{j}^{e}$ (in descending order), and (c) electron-positron 2-RDM $\Gamma^{e p}$ eigenvalues $g_{j}$ (in descending order) of a system containing a positron and $4+4$ electrons in various harmonic confinement strengths $\omega$.

the s-type states. The difference to the true many-body contact density is shown in Fig. 7(c), where it can be seen that the ansätze work moderately with $\omega=1,0.1$, but especially the product of the lowest natural orbitals fails to estimate the contact density in the strongly correlated regime.

\subsection{Larger systems and positronium formation}

We solved numerically the system containing a positron and four electrons of each spin in a harmonic trap, namely $1+4+4$ particles in total. Since there are as many particles as orbitals in the closed non-interacting energy shells, and assuming non-zero $\omega$, the ground state is unique. Moreover, only those many-body configurations that had a total noninteracting energy smaller than a certain cutoff were included in the many-body basis used in the calculation. The cutoff was chosen as $33[\hbar \omega]$ that resulted in a basis spanned by roughly $2 \times 10^{8}$ basis vectors, whereas the full basis without any cutoff procedure would be untractable, almost $10^{16}$. Such many-body basis cutoff schemes work well with large $\omega$, namely weakly correlated systems, since some of the correlations, such as simultaneous occupations of multiple particles in higher energy shells, are omitted.

The eigenvalues of the ground state 1-RDMs and the electron-positron 2-RDM are shown in Fig. 8. The single-particle picture of positrons survives even to low values of confinement strength $\omega$, as all the positron 1-RDM eigenvalues are close to 1 (occupied) or 0 (unoccupied). This implies that the positron could be treated rather accurately in a mean-field level, where the electrons only screen the potential felt by the positron, while not breaking the shell structure. That is, the system is too packed for Ps-like bound states to occur, which would require stronger correlations that are seen as partially filled orbitals. Even if there are only minor correlations between the positron and the rest of the system, the electron 1-RDM eigenvalues, shown in Fig. 8(b), imply correlations between the electrons, especially when the confinement strength $\omega$ is decreased. The 


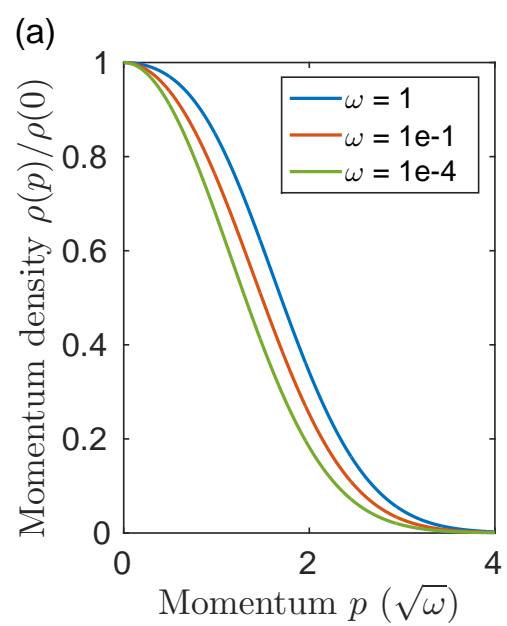

(d)

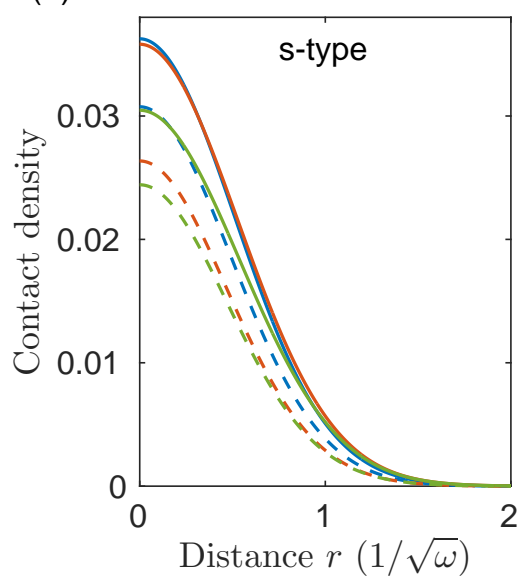

(b)

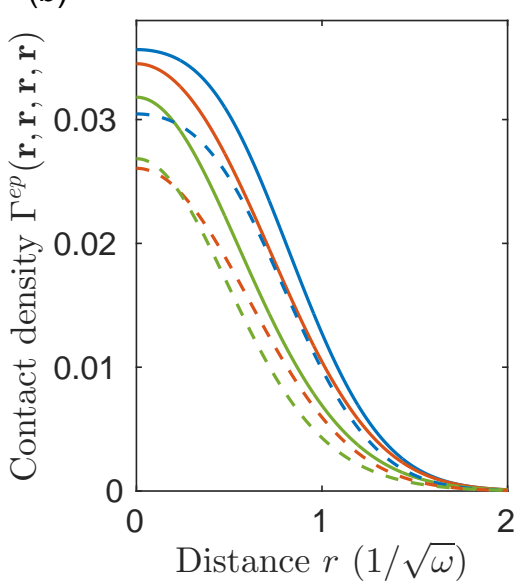

(e)

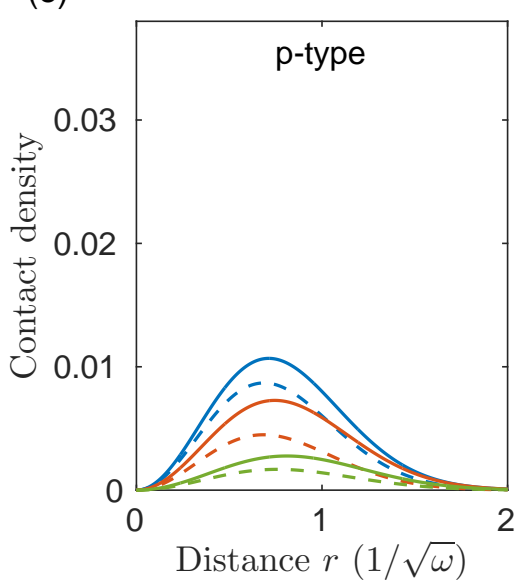

(c)

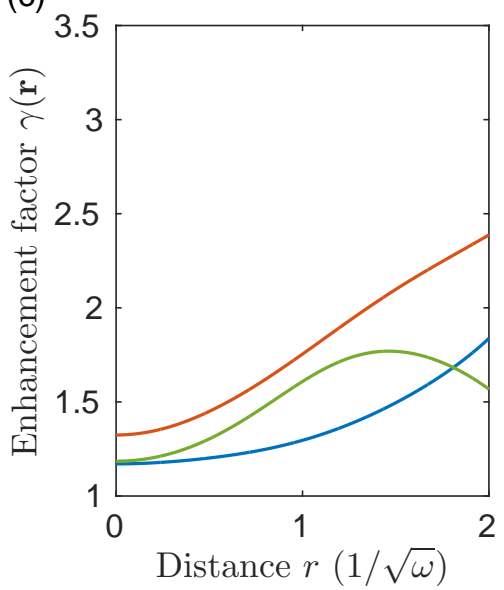

(f)

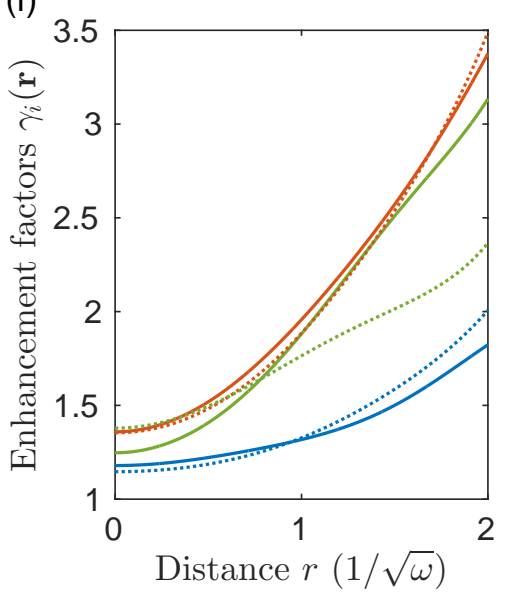

Figure 9. (a) Normalized momentum density of the annihilating electron-positron pairs $\rho(p) / \rho(0)$, (b) the total contact density (solid lines) and the contact density resulting in the independent particle approximation (dashed lines), and (c) the stateindependent enhancement factors $\gamma(\mathbf{r})$ of the $1+4+4$ system ground state in various confinement strengths $\omega$. (d) The contact densities of s-type states $\alpha_{0}$ (solid lines) and $\varphi_{0}^{e} \varphi_{0}^{p}$ (dashed lines), (e) the contact densities of the p-type degenerate states $\alpha_{i}$ (solid lines) and $\varphi_{i}^{e} \varphi_{0}^{p}$ (dashed lines) with $i=1,2,3$, and (f) the resulting shell-dependent enhancement factors for the s-type states (solid lines) and p-type states (dotted lines).

higher energy shells are also partially occupied by electrons. The electron-positron 2RDM eigenvalues $g_{j}$, shown in Fig. 8(c), are similar to those of the electron 1-RDM eigenvalues $n_{j}^{e}$. This can be explained by considering the limit of vanishing electronpositron correlations, such that the eigenvalues satisfy $g_{j}=n_{0}^{p} n_{j}^{e}$ and for the natural geminals $\alpha_{j}\left(\mathbf{r}_{p}, \mathbf{r}_{e}\right)=\varphi_{0}^{p}\left(\mathbf{r}_{p}\right) \varphi_{j}^{e}\left(\mathbf{r}_{e}\right)$, where the positron is assumed to be described by a single orbital $\varphi_{0}^{p}$ such that $n_{0}^{p}=1$.

The momentum density of annihilating electron-positron pairs in the $1+4+4$ system is shown in Fig. 9(a) with various values of confinement $\omega$. The Gaussian shape of the momentum distribution is not altered much by the confinement strength $\omega$. The many-body contact densities $\Gamma^{e p}(\mathbf{r}, \mathbf{r}, \mathbf{r}, \mathbf{r})$ are again compared to the contact densities 
in the independet particle approximation in Fig. 9(b). Even if the highest positron $1-\mathrm{RDM}$ eigenvalue is close to 1 , the independent contact density $n_{-}(\mathbf{r}) n_{+}(\mathbf{r})$ clearly underestimates the true contact densities, also in the least correlated case considered that is $\omega=1$. The resulting enhancement factors, defined as the ratio of $\Gamma^{e p}(\mathbf{r}, \mathbf{r}, \mathbf{r}, \mathbf{r})$ and $n_{-}(\mathbf{r}) n_{+}(\mathbf{r})$, are shown in $9(\mathrm{c})$. The effect of confinement $\omega$ is surprisingly small, as the enhancement factor curves are similar especially close to the trap center.

We evaluated the contact densities of the lowest s-type and p-type shells, namely summing the contact densities of the states with indices $j=0$ and $j=1,2,3$, respectively. The many-body contact densities are compared to the contact densities of the natural orbital product states in Fig. 9(d) and Fig. 9(e). The resulting shelldependent enhancement factors are shown in Fig. 9(f). The symmetry of the p-shell is clearly visible by the vanishing of the contact density at the harmonic trap center. Quite interestingly, the shell-dependent enhancement factors are slightly larger than the enhancement factors defined as the ratio of $\Gamma^{e p}(\mathbf{r}, \mathbf{r}, \mathbf{r}, \mathbf{r})$ and $n_{-}(\mathbf{r}) n_{+}(\mathbf{r})$. The main difference is that the geminal and orbital eigenvalues are not taken into account here, as they are not included in the definition of the state-dependent enhancement factors, whereas the full spectrum of the eigendecomposition is used to evaluate Eq. (15) and Eq. (17).

In open shell systems, on the other hand, the ground state manifold has typically more correlations, as there are multiple many-body configurations with similar weights. The natural geminals of electron-positron pairs are again the quantum states relevant to Ps formation and to related observables. In the general case, the ground state wave function can be written using the natural geminals as [43]

$\Psi\left(\mathbf{r}_{p}, \mathbf{r}_{e}, \mathbf{r}_{3}, \ldots, \mathbf{r}_{N}\right)=\sum_{i} c_{i} \alpha_{i}^{p e}\left(\mathbf{r}_{p}, \mathbf{r}_{e}\right) \beta_{i}\left(\mathbf{r}_{3}, \ldots \mathbf{r}_{N}\right)$,

where $\beta_{i}\left(\mathbf{r}_{3}, \ldots \mathbf{r}_{N}\right)$ are the $(N-2)$-particle natural states that are the eigenfunctions of the $(N-2)$-RDM, and $c_{i}$ are the complex coefficients for which $\left|c_{i}\right|^{2}$ matches the eigenvalues of both natural states. Curiously, the right-hand side is antisymmetric without the need of an explicit antisymmetrization, if the left hand side is antisymmetric. However, constructing such a linear combination without a priori knowledge of the ground state might be difficult, as one is faced with the $N$-representability problem. Furthermore, even in the case of several non-interacting electrons, there must be more than just a single term in the expansion, suggesting that multiple orthogonal geminals are needed in such an ansatz.

\section{Discussion and Conclusions}

Modeling of the pick-off annihilation properties of Ps in molecular materials is necessary in connection with the interpretation of experimental positron annihilation data. However, ab-initio models of Ps are computational heavy problems which presently cannot be addressed without certain degree of approximation. The simplifications done to the full problem will limit the accuracy of the predicted values. 
We have described a full-correlation Ps-positron potential for Ps interacting with He. It is characterized by a long range tail caused by the extremely low mass of Ps. We have also presented a scheme that for the first time includes full correlation Ps-positron potentials in atomistic models of a molecular soft material and is able to provide accurate distributions and pick-off annihilation rates. This scheme is able to describe the impact of the void size and it is sensitive to the electronic properties of the host material. It can address properties like the effect of the dispersion attraction, the shape of the void or the partial charges of the molecules [27].

We have shown how it can be implemented in a model of solid He which keeps the main characteristic of molecular materials, i.e., relatively weak molecular bonds and a HOMO-LUMO energy gap that prevents the capture of electrons by the host molecules. The pick-off annihilation rates and the localization energies in quasi-spherical voids fall into a common curve depending only on the size of the void, and independently of the lattice parameter of the crystal. Remarkably, the pick-off annihilation rate agrees with the prediction of the Tao-Eldrup model, once the layer parameter is adjusted for the low polarizability of He. The present results suggests that the layer parameter is larger as the size of the voids increases by the influence of the weak attractive dispersion. Partial charges present in molecular soft-matter can have a large impact in the annihilation properties (described in Zubiaga et al. [27]).

Accurate description of $\mathrm{Ps}$ in molecular materials will require developing appropriate electron and positron potentials that can be scaled for systems of any size. One pathway would be studying the generalization of effective positron potentials for larger molecular systems. Although this is difficult task for big molecules, already the knowledge gained from smaller model systems will guide to improve the description of Ps in those systems.

On a more conceptual level, we have found the concepts of reduced density matrices and their eigenfunctions such as the natural geminals useful for analyzing and understanding the nature of electron-positron correlations in fully interacting manybody systems. This theoretical framework enables a general and unique two-particle picture for the analysis of the correlation effects without the need to neglect any interactions or correlations present in the system.

\section{Acknowledgments}

M.E. acknowledges financial support from the Finnish Cultural Foundation. I.M. acknowledges financial support from the Academy of Finland (projects 285809 and 293932). We are grateful for the computational resources provided by Aalto Science-IT project and Finnish IT Center for Science (CSC).

\section{References}

[1] Stepanov S V and Byakov V M 2003 Principles and application of positron 6 positronium chemistry (World Scientific) p 117 
Modeling positronium beyond the single particle approximation

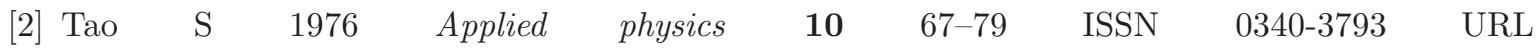
http://dx.doi.org/10.1007/BF00929530

[3] Mogensen O E 1995 Positron Annihilation in Chemistry (Springer-Verlag, Berlin)

[4] Brawley S J, Armitage S, Beale J, Leslie D E, Williams A I and Laricchia G 2010 Science 330789 (Preprint http://www.sciencemag.org/content/330/6005/789.full.pdf) URL http://www. sciencemag. org/content/330/6005/789. abstract

[5] Gidley D W, Frieze W E, Dull T L, Yee A F, Ryan E T and Ho H M 1999 Phys. Rev. B 60(8) R5157-R5160 URL http://link.aps.org/doi/10.1103/PhysRevB.60.R5157

[6] Liszkay L, Corbel C, Perez P, Desgardin P, Barthe M F, Ohdaira T, Suzuki R, Crivelli P, Gendotti U, Rubbia A, Etienne M and Walcarius A 2008 Applied Physics Letters 92063114 URL http://scitation.aip.org/content/aip/journal/apl/92/6/10.1063/1.2844888

[7] Nakanishi H and Ujihira Y 1982 The Journal of Physical Chemistry 86 4446-4450 URL http://dx.doi.org/10.1021/j100219a035

[8] Milina M, Mitchell S, Cooke D, Crivelli P and Pérez-Ramírez J 2015 Angewandte Chemie International Edition $\mathbf{5 4}$ 1591-1594 ISSN 1521-3773 URL http://dx.doi.org/10.1002/anie.201410016

[9] Rana U, Nambissan P M G, Malik S and Chakrabarti K 2014 Phys. Chem. Chem. Phys. 16(7) 3292-3298 URL http://dx.doi.org/10.1039/C3CP54032D

[10] Crivelli P, Cooke D, Barbiellini B, Brown B L, Feldblyum J I, Guo P, Gidley D W, Gerchow L and Matzger A J 2014 Phys. Rev. B 89(24) 241103 URL http://link.aps.org/doi/10.1103/PhysRevB.89.241103

[11] Uedono A, Suzuki R, Ohdaira T, Uozumi T, Ban M, Kyoto M, Tanigawa S and Mikado T 1998 Journal of Polymer Science Part B: Polymer Physics 36 2597-2605 ISSN 1099-0488 URL http://dx.doi.org/10.1002/(SICI) 1099-0488(199810) 36:14<2597: :AID-POLB12>3.0.CO;2-6

[12] Kobayashi Y, Kazama S, Inoue K, Toyama T, Nagai Y, Haraya K, Mohamed H F M, O'Rouke B E, Oshima N, Kinomura A and Suzuki R 2014 The Journal of Physical Chemistry B 118 6007-6014 pMID: 24815092 (Preprint http://dx.doi.org/10.1021/jp501706d) URL http://dx.doi.org/10.1021/jp501706d

[13] Sane P, Salonen E, Falck E, Repakova J, Tuomisto F, Holopainen J M and Vattulainen I 2009 The Journal of Physical Chemistry B 113 1810-1812 pMID: 19199698 (Preprint http://dx.doi.org/10.1021/jp809308j) URL http://dx.doi.org/10.1021/jp809308j

[14] Dong A W, Pascual-Izarra C, Pas S J, Hill A J, Boyd B J and Drummond C J 2009 The Journal of Physical Chemistry B 113 84-91 pMID: 19086790 (Preprint http://dx.doi.org/10.1021/jp805280r) URL http://dx.doi.org/10.1021/jp805280r

[15] Puska M J and Nieminen R M 1994 Rev. Mod. Phys. 66(3) 841-897 URL http://link.aps.org/doi/10.1103/RevModPhys.66.841

[16] Tuomisto F and Makkonen I 2013 Rev. Mod. Phys. 85(4) 1583-1631 URL http://link.aps.org/doi/10.1103/RevModPhys.85.1583

[17] Fabrikant I I and Gribakin G F 2014 Phys. Rev. Lett. 112(24) 243201 URL http://dx.doi.org/10.1103/PhysRevLett.112.243201

[18] Zubiaga A, Tuomisto F and Puska M J 2012 Phys. Rev. A 85(5) 052707 URL http://link.aps.org/doi/10.1103/PhysRevA.85.052707

[19] Tao S J 1972 The Journal of Chemical Physics 56 5499-5510 URL http://scitation.aip.org/content/aip/journal/jcp/56/11/10.1063/1.1677067

[20] Eldrup M, Lightbody D and Sherwood J 1981 Chemical Physics 6351 - 58 ISSN 0301-0104 URL http://www.sciencedirect.com/science/article/pii/0301010481803072

[21] Jasińska B, Kozioł A and Goworek T 1996 Journal of Radioanalytical and Nuclear Chemistry 210 617-623 ISSN 0236-5731 URL http://dx.doi.org/10.1007/BF02056403

[22] Dull T L, Frieze W E, Gidley* D W, Sun J N and Yee A F 2001 The Journal of Physical Chemistry B 105 4657-4662 (Preprint http://dx.doi.org/10.1021/jp004182v) URL http://dx.doi.org/10.1021/jp004182v 
Modeling positronium beyond the single particle approximation

[23] Goworek T, Jasińska B, Wawryszczuk J, Zaleski R and Suzuki T 2002 Chemical Physics $280295 \quad-\quad 307$ ISSN 0301-0104 URL http://www.sciencedirect.com/science/article/pii/S0301010402004913

[24] Wada K and Hyodo T 2013 Journal of Physics: Conference Series 443012003 ISSN 1742-6596 URL http://stacks.iop.org/1742-6596/443/i=1/a=012003

[25] Schmitz H and Müller-Plathe F 2000 The Journal of Chemical Physics 112 1040-1045 URL http://scitation.aip.org/content/aip/journal/jcp/112/2/10.1063/1.480627

[26] Saniz R, Barbiellini B, Platzman P M and Freeman A J 2007 Phys. Rev. Lett. 99(9) 096101 URL http://link.aps.org/doi/10.1103/PhysRevLett.99.096101

[27] Zubiaga A, Tuomisto F and Puska M J 2015 The Journal of Physical Chemistry B 119 1747-1755 pMID: 25523231 (Preprint http://dx.doi.org/10.1021/jp5106295) URL http://dx.doi.org/10.1021/jp5106295

[28] Varga $\mathrm{K}$ and Suzuki Y 1995 Phys. Rev. C $\mathbf{5 2 ( 6 )}$ 2885-2905 URL http://link.aps.org/doi/10.1103/PhysRevC.52.2885

[29] Schrader D M, Jacobsen F M, Frandsen N P and Mikkelsen U 1992 Phys. Rev. Lett. 69(1) 57-60 URL http://link.aps.org/doi/10.1103/PhysRevLett.69.57

[30] Ryzhikh G G, Mitroy J and Varga K 1998 Journal of Physics B: Atomic, Molecular and Optical Physics 313965 URL http://stacks.iop.org/0953-4075/31/i=17/a=019

[31] Mitroy J 2006 Phys. Rev. A 73(5) 054502 URL http://link. aps.org/doi/10.1103/PhysRevA.73. 054502

[32] Bubin S and Varga K 2011 Phys. Rev. A 84(1) 012509 URL http://link.aps.org/doi/10.1103/PhysRevA.84.012509

[33] Mitroy J 2010 J. At. Mol. Sci. 1 275-279

[34] Mitroy J and Ryzhikh G G 2001 Journal of Physics B: Atomic, Molecular and Optical Physics 34 2001 URL http://stacks.iop.org/0953-4075/34/i=10/a=313

[35] Mitroy J, Bromley M W J and Ryzhikh G G 2002 Journal of Physics B: Atomic, Molecular and Optical Physics 35 R81 URL http://stacks.iop.org/0953-4075/35/i=13/a=201

[36] Zubiaga A, Tuomisto F and Puska M J 2014 Phys. Rev. A 89(5) 052707 URL http://link.aps.org/doi/10.1103/PhysRevA.89.052707

[37] Zubiaga A, Tuomisto F and Puska M J 2013 Journal of Physics: Conference Series 443012004 URL http://stacks.iop.org/1742-6596/443/i=1/a=012004

[38] Seitsonen A P, Puska M J and Nieminen R M 1995 Phys. Rev. B 51(20) 14057-14061 URL http://link.aps.org/doi/10.1103/PhysRevB.51.14057

[39] Heiskanen M, Torsti T, Puska M J and Nieminen R M 2001 Phys. Rev. B 63(24) 245106 URL http://link.aps.org/doi/10.1103/PhysRevB.63.245106

[40] Glyde H R and Svensson E C 1987 Neutron Scattering, Part B Neutron Scattering in Condensed Matter Research (Academic Press, Waltham, Massachusetts)

[41] Rytsölä K, Vettenranta J and Hautojärvi P 1984 Journal of Physics B: Atomic and Molecular Physics 173359 URL http://stacks.iop.org/0022-3700/17/i=16/a=018

[42] Tang K T and Toennies J P 1984 The Journal of Chemical Physics 80 3726-3741 URL http://scitation.aip.org/content/aip/journal/jcp/80/8/10.1063/1.447150

[43] Coleman A J 1963 Rev. Mod. Phys. 35(3) 668-686 URL http://link.aps.org/doi/10.1103/RevModPhys.35.668

[44] Mazziotti D A 2012 Phys. Rev. Lett. 108(26) 263002 URL http://link.aps.org/doi/10.1103/PhysRevLett.108.263002

[45] Gilbert $\mathrm{T}$ L 1975 Phys. Rev. B 12(6) 2111-2120 URL http://link.aps.org/doi/10.1103/PhysRevB.12.2111

[46] Nagy A 2002 Phys. Rev. A 66(2) 022505 URL http://link.aps.org/doi/10.1103/PhysRevA.66.022505

[47] Tölö E and Harju A 2010 Phys. Rev. B 81(7) 075321 URL http://link.aps.org/doi/10.1103/PhysRevB.81.075321

[48] Green D G, Ludlow J A and Gribakin G F 2014 Phys. Rev. A 90(3) 032712 URL http://link.aps.org/doi/10.1103/PhysRevA.90.032712 
[49] Green D G and Gribakin G F 2015 Phys. Rev. Lett. 114(9) 093201 URL http://link.aps.org/doi/10.1103/PhysRevLett.114.093201

[50] Green D G and Gribakin G F arXiv 1502.08045 URL http://arxiv.org/abs/1502.08045

[51] Makkonen I, Ervasti M M, Siro T and Harju A 2014 Phys. Rev. B 89(4) 041105 URL http://link.aps.org/doi/10.1103/PhysRevB.89.041105 